\title{
Uniqueness of zero surface gravity SU(2) Einstein-Yang/ Mills black holes
}

\author{
J. A. Smoller and A. G. Wasserman \\ Department of Mathematics, University of Michigan, Ann Arbor, Michigan 48109-1003
}

(Received 18 September 1995; accepted for publication 10 November 1995)

In this paper we prove that the only spherically symmetric black hole solution to the SU(2) Einstein-Yang/Mills equations that has zero temperature at the event horizon is the extreme Reissner-Nordström solution. No assumptions are made on the signs of the metric coefficients, save that the metric has Lorentz signature. (C) 1996 American Institute of Physics. [S0022-2488(96)02803-2]

\section{INTRODUCTION}

Black holes having a degenerate horizon, that is, vanishing surface gravity, $\kappa=0$, are called extremal. In view of Hawking's celebrated formula (see Ref. 1),

$$
\kappa=2 \pi \tau
$$

where $\tau$ is the temperature at the horizon, this implies that such black holes have zero horizon temperature, and thus do not radiate; (however, see Refs. 2 and 3). The quantum mechanical stability of extremal black holes makes them very interesting objects, in various contexts. First, they are natural candidates for the final states of the evaporation process. Second, the scattering of quantum fields off extremal black holes can be described entirely within the semiclassical approximation, and this allows one to analyze the information loss in black hole evaporation without confronting the problem of unknown Plank-scale physics. ${ }^{4}$ Finally, it was suggested in Ref. 5 that extreme black holes resemble, in a certain sense, elementary particles-in fact, it was recently shown in Ref. 6 that extremal black holes can be identified with elementary string excitations.

In Ref. 7 it was shown that the only black-hole solution of the static, spherically symmetric, coupled Einstein-Yang/Mills (EYM) equations [with SU(2) gauge group], which has zero surface gravity, is the extreme Reissner-Nordstrom (ERN) solution. More precisely, if the Einstein metric is written in the form

$$
d s^{2}=-A(r) B(r)^{-2} d t^{2}+A^{-1}(r) d r^{2}+r^{2}\left(d \theta^{2}+\sin ^{2} \theta d \phi^{2}\right),
$$

and the SU(2) Yang/Mills field is (cf. Refs. 7-10)

$$
F=w^{\prime}(r) \tau_{1} d r \wedge r d \theta+w^{\prime}(r) \tau_{2} d r \wedge(\sin \theta d \phi)-\left(1-w^{2}(r)\right) \tau_{3} d \theta \wedge(\sin \theta d \phi)
$$

where $\tau_{1}, \tau_{2}, \tau_{3}$ form a basis for the Lie algebra $\mathrm{su}(2)$, then if

$$
\lim _{r \searrow \bar{r}} A(r)=0=\lim _{r \searrow \bar{r}} A^{\prime}(r), \quad A(r) \geqslant 0, \text { for } r>\bar{r}
$$

the metric must be the ERN metric; namely $A(r)=[(r-1) / r]^{2}, B(r) \equiv 1, w(r) \equiv 0$, and the YangMills curvature 2-form takes values in the Lie algebra $\mathrm{u}(1)$. It was also proved in Ref. 7 that for the metric (1.1), the surface gravity $\kappa=0$ if and only if $A^{\prime}$ vanishes at the black hole horizon $\bar{r}$.

In this paper we shall strengthen the above result, so as to also apply to the interior of a black hole. Namely, we will prove that if $(A, w)$ is a smooth solution of the EYM equations, defined for $r>\bar{r}$, such that $A$ is positive for some large $r$, and if 


$$
\lim _{r \searrow \bar{r}} A(r)=0=\lim _{r \searrow \bar{r}} A^{\prime}(r),
$$

then again the metric (1.1) is the ERN metric, $w(r) \equiv 0$, and the Yang/Mills curvature 2-form lies in $\mathrm{u}(1)$; cf. Theorem 3.1 The proof of this result is much more difficult than the proof in Ref. 7, because $A(r)$ is not assumed to be positive for $r>\bar{r}$.

Finally, we remark that it was proved in Ref. 10; (also see Ref. 11), that if $A(\bar{r})=0$ and $A^{\prime}(\bar{r}) \neq 0$, then the singularity in the metric at $r=\bar{r}$ can be transformed away by a "Kruskal-like" change of coordinates in which the YM field remains well behaved. Moreover, it was proved in Ref. 12 that for the ERN solution, the metric singularity at $r=\bar{r}$ can also be transformed away. It thus follows from our result here that for any SU(2) spherically symmetric EYM black hole solution with event horizon at $r=\bar{r}>0$, the singularity in the metric at $r=\bar{r}$ can be transformed away by a change of coordinates, whereby the YM field remains well behaved.

\section{PRELIMINARIES}

As discussed elsewhere, (cf. Refs. 8 and 9), the static, spherically symmetric EYM equations, with gauge group $\mathrm{SU}(2)$ can be written in the form

$$
\begin{gathered}
r A^{\prime}+\left(1+2 w^{\prime 2}\right) A=1-\frac{u^{2}}{r^{2}}, \\
r^{2} A w^{\prime \prime}+\left[r(1-A)-\frac{u^{2}}{r}\right] w^{\prime}+w\left(1-w^{2}\right)=0, \\
\frac{B^{\prime}}{B}=\frac{2 w^{\prime 2}}{r},
\end{gathered}
$$

where

$$
u(r)=1-w^{2}(r) .
$$

Since (2.1) and (2.2) do not involve $B$, we can use these to obtain $A$ and $w$, and then use (2.3) to find $B$. Here $w(r)$ is the connection coefficient that determines the Yang-Mills curvature 2-form; see Refs. 8 and 9. If we write

$$
\Phi(A, w, r)=r(1-A)-\frac{u^{2}}{r},
$$

then (2.1) and (2.2) can be written in the more compact form

$$
\begin{gathered}
r A^{\prime}+2 w^{\prime 2} A=\Phi / r, \\
r^{2} A w^{\prime \prime}+\Phi w^{\prime}+w\left(1-w^{2}\right)=0 .
\end{gathered}
$$

If $(A(r), w(r))$ is a specific solution of (2.1),(2.2), then we write $\Phi(r)=\Phi(A(r), w(r), r)$.

\section{THE THEOREM}

In this section we shall prove the following theorem.

Theorem 1: Let $(A(r), w(r))$ be a smooth solution of (2.1) and (2.2), satisfying

$$
\lim _{r \searrow \bar{r}} A(r)=0=\lim _{r \searrow \bar{r}} A^{\prime}(r),
$$




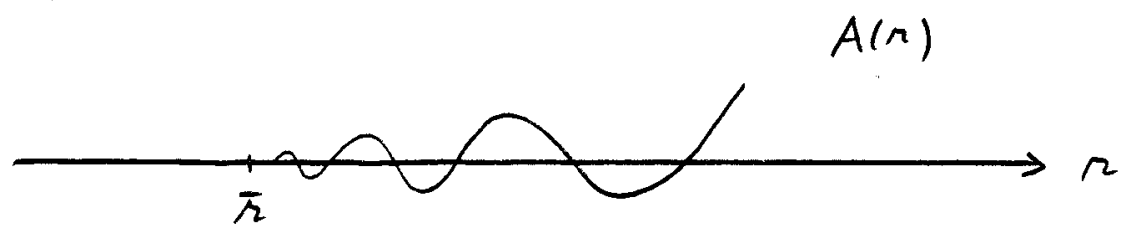

FIG. 1. A oscillates.

for some $\bar{r} \geqslant 0$, and assume $A\left(r_{1}\right)>0$ for some $r_{1}>\max (\bar{r}, 1)$. Then $(A, w)$ is the extreme ReissnerNordström (ERN) solution; namely,

$$
A(r)=\left(\frac{r-1}{r}\right)^{2}, \quad w(r) \equiv 0 .
$$

\section{Remarks:}

(1) If (3.2) holds, then from (2.3) we have $B(r) \equiv 1$.

(2) Theorem 1 was proved in Ref. 7 under the additional hypothesis that $A(r) \geqslant 0$ for $r>\bar{r}$. We show here that the theorem is still true under the far weaker hypothesis $A\left(r_{1}\right)>0$ for some $r_{1}>\max (1, \bar{r})$.

Proof of Theorem 1: There are three cases to consider; namely, for $\bar{r} \geqslant 0$, the following occurs.

Case (i). There is a sequence $r_{n} \searrow \bar{r}$ such that

$$
(-1)^{n} A\left(r_{n}\right)>0 .
$$

In this case we say that $A$ oscillates; cf. Fig. 1.

Case (ii). $A(r)<0$ for $r>\bar{r}, r$ near $\bar{r}$; cf. Fig. 2.

Case (iii). $A(r)>0$ for $r>\bar{r}, r$ near $\bar{r}$.

As mentioned above, a proof of the Theorem in case (iii) was given in Ref. 7, under the additional hypothesis that $A(r) \geqslant 0$ for $r>\bar{r}$.

We shall prove that neither of the cases (i) or (ii) can occur, and that if case (iii) occurs, the solution is the ERN solution. The proof is further divided into two subcases; namely either the solution $(A, w)$ is "smooth up to the boundary;" i.e., $(A, w) \in\left(C^{1} \times C^{2}\right)[\bar{r}, \bar{r}+\epsilon)$ for some $\epsilon>0$, or $(A, w)$ is not smooth at $\bar{r}$. The following proposition is subsumed by Theorem 1 . The simple proof is given here in order to demonstrate that the difficulties occur when neither $A$ nor $w$ is assumed to be smooth at $\bar{r}$.

Proposition 2: Suppose that $(A, w) \in\left(C^{1} \times C^{2}\right)[\bar{r}, \bar{r}+\epsilon)$ for some $\epsilon>0$. If (3.1) holds, then $\bar{r}=1$, and the solution is the ERN solution (3.2).

Before giving the proof, we shall need a preliminary result.

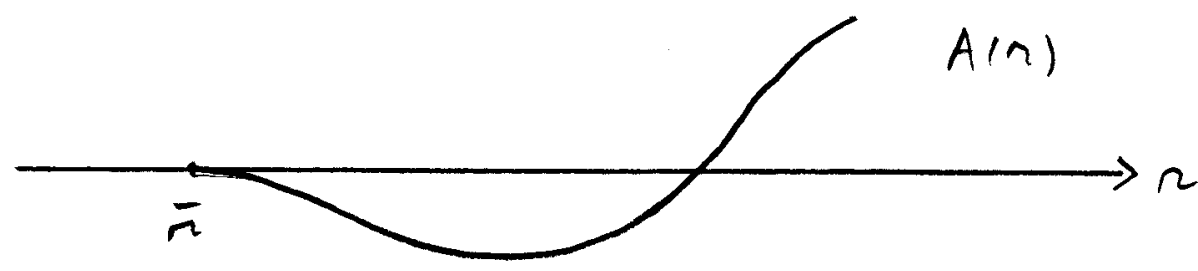

FIG. 2. $A<0$ near $\bar{r}$. 
Lemma 3: If $(A, w)$ is a solution of the EYM equations defined in an open interval about $r_{0}$, and if we have $w^{2}\left(r_{0}\right) \leqslant 1, A^{\prime}\left(r_{0}\right)=0$ and $A\left(r_{0}\right)<0$, then $r_{0}<1$.

Proof: If $r_{0} \geqslant 1$, then from (2.1) we obtain the contradiction

$$
0>\left(1+2 w^{\prime 2}\left(r_{0}\right)\right) A\left(r_{0}\right)=1-\frac{u^{2}\left(r_{0}\right)}{r_{0}^{2}} \geqslant 0 .
$$

Proof of Proposition 2: If $\bar{r}=0$, then (as in Ref. 10) expanding $A$ and $w$ in Taylor polynomials gives

$$
\begin{aligned}
& A(r)=A_{0}+A_{1} r+O\left(r^{2}\right), \\
& w(r)=w_{0}+w_{1} r+O\left(r^{2}\right),
\end{aligned}
$$

and we easily obtain from (2.1) and (2.2) that $A_{0}=1$. Thus, $A(0)=1$ and this violates (3.1). If $\bar{r}>0$, then from (2.6) we see $\Phi(\bar{r})=0$ so (2.5) gives $\bar{r}^{2}=\bar{u}^{2}$, where

$$
\bar{u}=1-\bar{w}^{2}, \quad \bar{w}=\bar{w}(\bar{r}) .
$$

From (2.7) we conclude $\bar{u} \bar{w}=0$, so since $\bar{r}>0$, we obtain $\bar{w}=0$, and thus $\bar{r}=1$. Now $A\left(r_{1}\right)>0$, $A(1)=0$, so $\min A(r)$ in the interval $\left[1, r_{1}\right]$ cannot be negative, in view of Lemma 3 ; hence $A(r) \geqslant 0$ on $1 \leqslant r \leqslant r_{1}$. To invoke the results of Ref. 7, we must show that $A(r) \geqslant 0$ for all $r>1$. To do this, suppose that $r_{2}$ was the first zero of $A, r_{2}>r_{1}$. If $w^{2}\left(r_{2}\right) \leqslant 1$, then from $(2.1)$ we find $A^{\prime}\left(r_{2}\right)>0$, so $A(r)<0$ for some $r<r_{2}, r$ near $r_{2}$, and this is impossible. If $w^{2}\left(r_{2}\right)>1$, then $w^{2}\left(r_{3}\right)>1$, and $A\left(r_{3}\right)>0$ for some $r_{3}<r_{2}, r_{3}$ near $r_{2}$. If $\left(w w^{\prime}\right)\left(r_{3}\right)>0$, it was shown in Ref. 10, Proposition 2.2, that $w^{\prime}$ tends to infinity for some $r>r_{3}$, thereby violating the smoothness assumption. If $\left(w w^{\prime}\right)\left(r_{3}\right)<0$, it was shown in Ref. 10, Proposition 2.3, that $A(\bar{r})>0$, thereby violating (3.1). If $\left(w w^{\prime}\right)\left(r_{3}\right)=0$, then $w^{\prime}\left(r_{3}\right)=0$, so (2.2) implies the contradiction $(u w)\left(r_{3}\right)=0$. Thus $A(r)>0$ if $r>r_{1}$, so $A(r) \geqslant 0$ if $r>\bar{r}$; hence the results of Ref. 7 apply to show $(A, w)$ is the ERN solution.

Remark: One case in which Proposition 2 applies is the following; namely, suppose that $(A(r), w(r))$ is the solution of (2.6) and (2.7), defined for $r>\bar{r}$, where $A(\bar{r})=0$, and $A(r)>0$ for $r>\bar{r}, r$ near $\bar{r}$. Then by Ref. 10, Theorems 3.4 and 3.7, the solution can be extended to be smooth at $\bar{r}$, if $\bar{r}>0$, and if $\bar{r}=0, A(0)=1$; hence Proposition 2 applies. Thus, in proving Theorem 1, we may assume that either case (i) or case (ii), above, hold. That is, we may assume that either there is a sequence $r_{n} \backslash \bar{r}$ such that (3.3) holds, or else that $A(r)<0$ for $r$ near $\bar{r}, r>\bar{r}$.

In what follows, we shall assume only that

$$
(A, w) \in\left(C^{1} \times C^{2}\right)(\bar{r}, \bar{r}+\epsilon),
$$

for some $\epsilon>0$; this case is far more difficult.

Notes. (1) We do not assume that $A$ or $w$ is smooth at $\bar{r}$, nor do we assume that our solution is regular, as in Ref. 10.

(2) The proof given in this paper is considerably more difficult than that in Ref. 10 because since we allow $A$ to change signs for $r$ near $\bar{r}$, the curve $\left(w(r), w^{\prime}(r)\right)$ can a priori be "all over" the $w-w^{\prime}$ plane. That is, both $A$ and $w$ can oscillate unboundedly and $w^{\prime}$ can be unbounded. We shall, in fact, show that none of the above can occur; this will require that we "systematically" rule out all such pathological behavior.

(3) In what follows, we assume that $(A, w)$ is not the ERN solution, and we shall prove that (3.1) leads to a contradiction.

Proof of Theorem 1: We begin with the following lemma.

Lemma 4: There does not exist a sequence $r_{n} \searrow \bar{r}$ satisfying $w\left(r_{n}\right)^{2}=1$. 
Proof: Suppose that the contrary holds. Then, by passing to a subsequence if necessary, we may assume, without loss of generality, that $w\left(r_{n}\right)=1$, and $w^{\prime}\left(r_{n}\right) \geqslant 0$, for $n=1,2, \ldots$. We claim that for each $n, A\left(r_{n}\right) \leqslant 0$. To see this, suppose that $A\left(r_{n}\right)>0$. Then, if $w^{\prime}\left(r_{n}\right)=0$, it follows by uniqueness that $w(r) \equiv 1$ and $A(r)=1+c / r$ for some constant $c$. This violates (1.2). Thus, we may assume that $w^{\prime}\left(r_{n}\right)>0$. It follows that the orbit enters the region $w>1, w^{\prime}>0$, for $r>r_{n}, r$ near $r_{n}$, with $A(r)>0$. From Ref. 10, Proposition 2.2, it follows that $w^{\prime}$ tends to infinity for some $r>\bar{r}$, so the solution cannot be smooth. This contradiction proves our claim; i.e. $A\left(r_{n}\right)>0$. Now, since $u\left(r_{n}\right)=0,(2.1)$ gives

$$
r_{n} A^{\prime}\left(r_{n}\right)+A\left(r_{n}\right)=-2\left(A w^{\prime 2}\right)\left(r_{n}\right)+1,
$$

and since $A\left(r_{n}\right) \leqslant 0$, we have

$$
r_{n} A^{\prime}\left(r_{n}\right)+A\left(r_{n}\right) \geqslant 1
$$

But this cannot hold for large $n$, in view of (3.1).

Corollary 5: There is an $\tilde{r}>\bar{r}$ such that $w^{2}(r) \neq 1$ if $\bar{r}<r<\tilde{r}$.

In view of this corollary, we may assume that precisely one of the following holds:

$$
\begin{gathered}
w(r)>1, \quad \text { if } \bar{r}<r<\tilde{r}, \\
w(r)<-1, \quad \text { if } \bar{r}<r<\tilde{r},
\end{gathered}
$$

or

$$
-1<w(r)<1 \text {, if } \bar{r}<r<\tilde{r} .
$$

In order to consider these cases, we shall need some preliminary results. We begin by noting that from (2.1), we have

$$
r A^{\prime}+A+2 A w^{\prime 2}=1-\frac{u^{2}}{r^{2}} \leqslant 1
$$

Also, given any $\delta>0$, (3.1) shows that for $r$ near $\bar{r}, r A^{\prime}(r)+A(r)>-\delta$, and so from (3.8),

$$
2 A w^{\prime 2} \leqslant 1+\delta
$$

Thus we have the following.

Lemma 6: There is an $\epsilon>0$ such that

$$
A(r) w^{\prime 2}(r) \leqslant 1, \quad \text { if } \bar{r}<r<\bar{r}+\epsilon .
$$

Thus $A w^{\prime 2}$ is bounded from above if $r$ is near $\bar{r}$. Our first goal is the show that $A w^{\prime 2}$ is bounded (Proposition 10); as a first step in this direction we have the following.

Lemma 7: Let $\bar{r}>0$, and assume that $w(r)$ is bounded for $r$ near $\bar{r}$. Then $\left(A w^{\prime 2}\right)(r)$ is bounded for $r$ near $\bar{r}$.

Proof: Assume that the result is false. In view of (3.10), we may assume that there is a sequence $r_{n} \searrow \bar{r}$ such that $\left(A w^{\prime 2}\right)\left(r_{n}\right) \rightarrow-\infty$. But from (2.1), we see that if $\bar{r}>0$ and $w$ is bounded, it follows that $A w^{\prime 2}$ is bounded in view of (3.1).

Lemma 8: Let $\bar{r} \geqslant 0$, and assume that there is an $\epsilon>0$ such that if $r$ is close to $\bar{r}$,

$$
\left(A w^{\prime 2}\right)(r) \leqslant-\frac{1}{2}-\epsilon
$$


Then $A w^{\prime 2}$ has a negative limit at $\bar{r}$ (which may equal $-\infty$ ), $A(r)$ is negative for $r$ near $\bar{r}$, and $w^{\prime 2}(r) \rightarrow \infty$ as $r \backslash \bar{r}$.

Proof: Let

$$
f=A w^{\prime 2}
$$

then (cf. Refs. 9 and 10) $f$ satisfies the equation

$$
r f^{\prime}+\left(2 f+\frac{\Phi}{r}\right) w^{\prime 2}+2 \frac{u}{r} w w^{\prime}=0
$$

Since $A(r) \rightarrow 0$, as $r \searrow \bar{r}$, we see $w^{\prime 2}(r) \rightarrow \infty$. Also, for $r$ near $\bar{r}$,

$$
2 f+\frac{\Phi}{r}=2 f+1-A-\frac{u^{2}}{r^{2}} \leqslant-2 \epsilon-A-\frac{u^{2}}{r^{2}}<-\epsilon-\frac{u^{2}}{r^{2}} \leqslant-\epsilon .
$$

We shall now show that

$$
f^{\prime}(r)>0, \text { if } r \text { is near } \bar{r} \text {. }
$$

To do this, we only consider those $r$ for which $\bar{r}<r<\tilde{r}$ [c.f. (3.5)-(3.7)].

Suppose first that for all such $r$, the sequence $\left\{w^{2}(r)\right\}$ is bounded. Then from (3.14), we have, at such $r$,

$$
\left(2 f+\frac{\Phi}{r}\right) w^{\prime 2}+2 \frac{u}{r} w w^{\prime}<-\epsilon w^{\prime 2}-\frac{u^{2}}{r^{2}} w^{\prime 2}+\frac{2 u}{r} w w^{\prime} .
$$

If $(u / r) w^{\prime} \rightarrow 0$, as $r \searrow \bar{r}$, then (3.14) shows that $(2 f+\Phi / r) w^{\prime 2}+(2 u / r) w w^{\prime}<0$, so from (3.13) we conclude that (3.15) holds. On the other hand, if $\lim _{r \backslash \bar{r}}\left|(u / r) w^{\prime}\right|>0$, then as $\{w(r)\}$ is bounded and $\left|w^{\prime}(r)\right| \rightarrow \infty$, we see from (3.16) that (3.15) holds. Thus, (3.15) holds if $\left\{w^{2}(r)\right\}$ is bounded near $\bar{r}$.

Suppose now that $w^{2}\left(r_{n}\right) \rightarrow \infty$ for some sequence $r_{n} \searrow \bar{r}$; we shall show that for large $n$, (3.15) holds. Thus, if $w^{2}\left(r_{n}\right) \rightarrow \infty$, we have, at $r=r_{n}$,

$$
\begin{aligned}
-\frac{u^{2}}{r_{n}^{2}} w^{\prime 2}+2 \frac{u}{r_{n}} w w^{\prime} & =\frac{-u}{r_{n}^{2}}\left[u w^{\prime 2}-2 w w^{\prime} r_{n}\right] \\
& =\frac{-u}{r_{n}^{2}}\left[\left(1-w^{2}\right) w^{\prime 2}-2 w w^{\prime} r_{n}\right] \\
& <\frac{-u}{r_{n}^{2}}\left[-\frac{w^{2}}{2} w^{\prime 2}-2 w w^{\prime} r_{n}\right]
\end{aligned}
$$

if $n$ is large. Let $x_{n}=w\left(r_{n}\right) w^{\prime}\left(r_{n}\right)$; then $x_{n}^{2} \rightarrow \infty$, and if $h\left(x_{n}\right)=-x_{n}^{2} / 2-2 x_{n} r_{n}$, then if $x_{n} \rightarrow+\infty$, $h\left(x_{n}\right)=\left(x_{n} / 2\right)\left(-x_{n}+4 r_{n}\right) \rightarrow-\infty$ [since $u\left(r_{n}\right) \rightarrow-\infty$ ], while if $x_{n_{k}} \rightarrow-\infty$ for some subsequence $\left\{n_{k}\right\}$, then $h\left(x_{n_{k}}\right)=\left(-x_{n_{k}} / 2\right)\left(x_{n_{k}}-4 r_{n_{k}}\right) \rightarrow-\infty$. Thus, (3.17) and (3.16) show that (3.15) holds, at $x_{n_{k}}$, so that (3.15) is valid. Thus $\lim _{r \backslash \bar{r}} f(r)$ exists and is $\leqslant-\frac{1}{2}$, so $A(r)$ is negative for $r$ near $\bar{r}$, and $w^{\prime 2}(r) \rightarrow \infty$ as $r \backslash \bar{r}$. This completes the proof of Lemma 8 .

Lemma 9: Let $\bar{r}=0$; then $\left(A w^{\prime 2}\right)(r)$ is bounded for $r$ near 0 .

Proof: Suppose $A w^{\prime 2}$ is not bounded near $r=0$. Then, in view of Lemma $6, A w^{\prime 2}$ is not bounded from below, so we can find a sequence $r_{n} \searrow 0$ such that $\left(A w^{\prime 2}\right)\left(r_{n}\right) \leqslant-\frac{1}{2}-\epsilon$, for some 


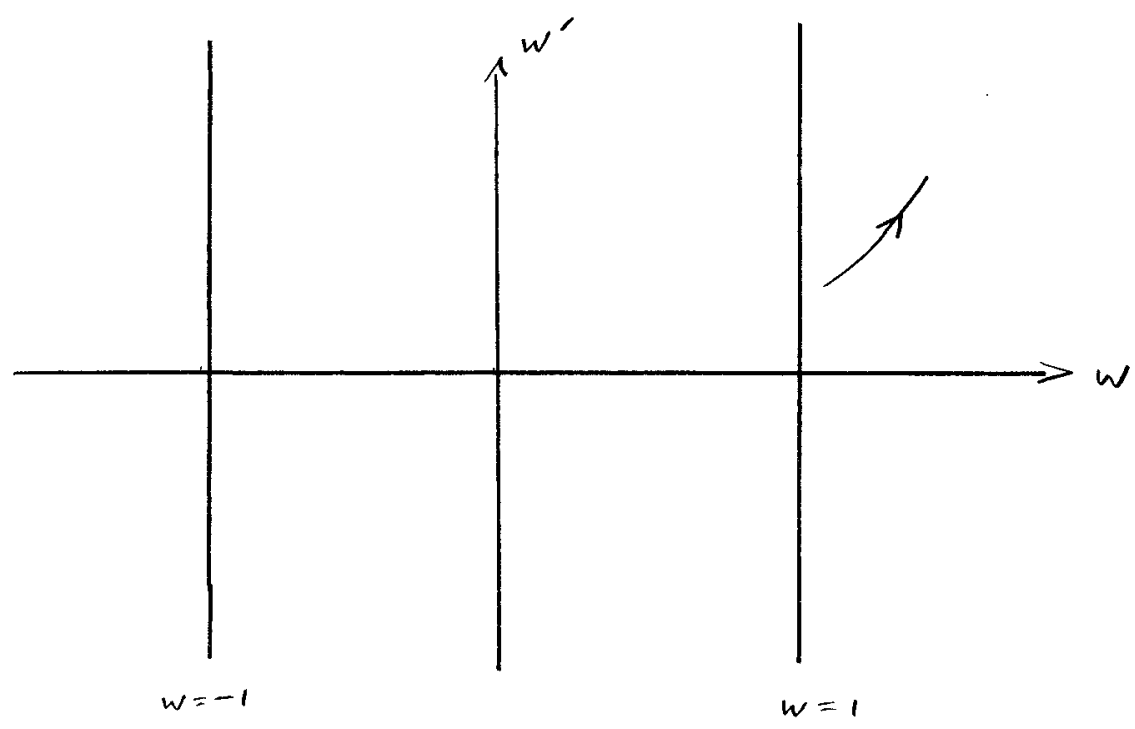

FIG. 3. $w$ is bounded near $r=0$.

$\epsilon>0$. By the last lemma, $A w^{\prime 2}$ has a negative limit at $r=0$, and as $A w^{\prime 2}$ is unbounded near $r=0$, we see that $\left(A w^{\prime 2}\right)(r) \rightarrow-\infty$ as $r \searrow 0$. Thus, from the last lemma, we have

$$
A(r)<0, \text { for } r \text { near } 0,
$$

$w^{\prime}(r)$ is of one sign for $r$ near 0 ,

$$
\lim _{r \searrow 0} w^{\prime 2}(r)=\infty .
$$

Next, from $(2.1) \lim _{r \backslash 0}\left[u^{2} / r^{2}+2\left(A w^{\prime 2}\right)(r)\right]=1$, and as $A w^{\prime 2} \rightarrow-\infty$, we see

$$
\lim _{r \searrow 0} \frac{u^{2}(r)}{r^{2}}=\infty .
$$

Thus $r^{2} / u^{2} \rightarrow 0$ as $r \searrow 0$, so

$$
\lim _{r \searrow 0} \frac{2\left(A w^{\prime 2}\right)(r)}{u^{2} / r^{2}}=-1 .
$$

Since (3.21) holds, we have

$$
-\frac{\Phi}{r}=-1+A+\frac{u^{2}}{r^{2}}>\frac{u^{2}}{2 r^{2}} \text { for } r \text { near } 0 .
$$

Now, in view of (3.19) and (3.20), either $\lim _{r \searrow 0} w^{\prime}(r)=+\infty$ or $\lim _{r \searrow 0} w^{\prime}(r)=-\infty$. Then, in either case $\lim _{r \backslash 0} w(r)$ exists. Suppose first that $\lim _{r \backslash 0} w^{\prime}(r)=\infty$ [the case where $w^{\prime}(r) \rightarrow-\infty$ will be discussed below]. We consider $r$ in the range $0=\bar{r}<r<\tilde{r}$; cf. (3.5)-(3.7). Then there are three possibilities: $w(r)$ is bounded, $\lim _{r \backslash 0} w(r)=-\infty$, or $\lim _{r \backslash 0} w(r)$ $=+\infty$. Note first that since $w^{\prime}(r) \rightarrow \infty$, if $w(r) \rightarrow+\infty$, then $w$ is bounded near 0 ; cf. Fig. 3. Thus 
we shall suppose that

$$
\lim _{r \searrow 0} w^{\prime}(r)=\infty,
$$

and either

$$
\lim _{r \searrow 0} w(r)=-\infty,
$$

or

$$
w(r) \text { is bounded near } r=0 \text {; }
$$

we shall obtain a contradiction in both cases.

Now for $r$ near 0 , we have, from (2.7) and (3.23),

$$
r A w^{\prime \prime}=\frac{-\Phi}{r} w^{\prime}-\frac{u}{r} w>\frac{u^{2}}{2 r^{2}} w^{\prime}-\frac{u}{r} w \geqslant \frac{1}{4} \frac{u^{2}}{r^{2}} w^{\prime},
$$

because (3.25) or (3.26) holds. That is, if (3.26) holds, then (3.21) implies

$$
\frac{u^{2}}{4 r^{2}} w^{\prime}-\frac{u}{r} w=\frac{u}{4 r}\left[\frac{u}{r} w^{\prime}-4 w\right]>0, \quad \text { if } r \text { is near } 0
$$

while if (3.25) holds, then since $w=o(u)$,

$$
\frac{u^{2}}{4 r^{2}} w^{\prime}-\frac{u}{r} w=\frac{u}{4 r}\left[\frac{\left(1-w^{2}\right)}{r} w^{\prime}-4 w\right]>0 .
$$

Thus, for $r$ near $0,(3.27)$ and (3.22) give, for $r$ near 0 ,

$$
-w^{\prime \prime} \geqslant \frac{1}{4} \frac{u^{2}}{\left(-A r^{2}\right)} w^{\prime}=\frac{1}{4} \frac{u^{2}}{r^{2}}\left(\frac{1}{-A w^{\prime 2}}\right) \frac{w^{\prime 3}}{r} \geqslant \frac{c^{2}}{r} w^{\prime 3},
$$

where $c$ is a positive constant. Now let $0<t<s$, where $s$ is near 0 . Then, from (3.28) we obtain

$$
\frac{1}{2}\left[\frac{1}{w^{\prime 2}(t)}-\frac{1}{w^{\prime 2}(s)}\right]=-\left.\frac{1}{2} \frac{1}{w^{\prime 2}(r)}\right|_{t} ^{s}=\int_{t}^{s} \frac{w^{\prime \prime}}{w^{\prime 2}} d r \geqslant c^{2} \int_{t}^{s} \frac{d r}{r}=c^{2} \ln \frac{s}{t},
$$

so that

$$
\frac{1}{2}\left[\frac{1}{w^{\prime 2}(t)}-\frac{1}{w^{\prime 2}(s)}\right] \geqslant c^{2} \ln \frac{s}{t}
$$

Now let $t \rightarrow 0$; then the left side of (3.28) is bounded [because of (3.20)], but the right side tends to $\infty$. This contradiction shows that the lemma holds if (3.24), and either (3.25) or (3.26) holds.

Now suppose that

$$
\lim _{r \searrow 0} w^{\prime}(r)=-\infty,
$$

and either 


$$
\lim _{r \searrow 0} w(r)=\infty,
$$

or (3.25) holds; we shall indicate how to obtain a contradiction. [It is easy to see that if (3.29) holds then $\lim _{r \backslash \bar{r}} w(r) \neq-\infty$.] In this case we obtain, from (2.7), (3.23), and (3.29),

$$
r A w^{\prime \prime} \leqslant \frac{u^{2}}{2 r^{2}} w^{\prime}-\frac{u}{r} w \leqslant \frac{u^{2}}{4 r^{2}} w^{\prime},
$$

if $r$ is near 0 , so that, using (3.22),

$$
-w^{\prime \prime} \leqslant \frac{1}{4} \frac{u^{2}}{\left(-A r^{3}\right)} w^{\prime}=\frac{1}{4}\left[\frac{u^{2} / r^{2}}{-A w^{\prime 2}}\right] \frac{w^{\prime 3}}{r} \leqslant c^{2} \frac{w^{\prime 3}}{r},
$$

so $w^{\prime \prime} \geqslant\left(-c^{2} / r\right) w^{\prime 3}$, and thus $\left(-w^{\prime \prime} / w^{\prime 3}\right) \geqslant c^{2} / r$. If we again integrate from $t$ to $s$, we get

$$
\left.\frac{1}{2} \frac{1}{w^{\prime 2}(r)}\right|_{t} ^{s} \geqslant c^{2} \lim \frac{s}{t},
$$

and letting $t \rightarrow 0$ gives a contradiction, as before. This completes the proof of Lemma 9 .

We next have the following.

Proposition 10: $\left(A w^{\prime 2}\right)(r)$ is bounded if $r$ is near $\bar{r}$.

Proof: From Lemma 9, we may assume that $\bar{r}>0$. Now choose $r$ such that $\bar{r}<r<\tilde{r}$, where $\tilde{r}$ is defined in Corollary 5. Thus, as we have shown above, (3.5)-(3.7) are valid. Now if $w(r)$ is bounded, then Lemma 7 implies that $A w^{\prime 2}$ is bounded near $\bar{r}$. Thus, we may assume that $w$ is unbounded near $\bar{r}$, so that for $\bar{r}<r<\tilde{r}$, either $w(r)>1$ or $w(r)<-1$. Since the proofs are similar in both cases, we shall restrict attention to the case $w(r)>1$ for $\bar{r}<r<\tilde{r}$.

Thus, assume that $w$ is unbounded near $\bar{r}$, and $w(r)>1$ for $\bar{r}<r<\tilde{r}$. Now suppose that $A w^{\prime 2}$ is not bounded for $r$ near $\bar{r}$. Then, as in the proof of Lemma $9, \lim _{r \backslash \bar{r}}\left(A w^{\prime 2}\right)(r)=-\infty$, and (3.18)-(3.20) are valid. Thus, from (3.19), we conclude that $\lim _{r \backslash \bar{r}} w(r)$ exists; hence

$$
\lim _{r \searrow \bar{r}} w(r)=+\infty .
$$

Also, the orbit cannot stay in the region $w^{\prime}>0$ for $r$ near $\bar{r}$, for otherwise it would follow that $w$ is bounded near $\bar{r}$; cf. Fig. 3. Thus the orbit enters the region $w^{\prime}<0$, and in view of (3.19), we may assume that $w^{\prime}(r)<0$ if $r$ is near $\bar{r}$; cf. Fig. 4. In view of (3.19) we have

$$
\lim _{r \searrow \bar{r}} w^{\prime}(r)=-\infty .
$$

From (2.7) we can write

$$
A w^{\prime \prime} w^{\prime 2}+\frac{\Phi}{r^{2}} w^{\prime 3}+\frac{u w}{r^{2}} w^{\prime 2}=0 .
$$

Now, from (2.1),

$$
r A^{\prime}+A+2 A w^{\prime 2}=1-\frac{u^{2}}{r^{2}}
$$

so that for $r$ near $\bar{r}$, since $\bar{r}>0$,

$$
\left(A w^{\prime 2}\right) \text { is well approximated by }-c_{1}^{2} w^{4},
$$




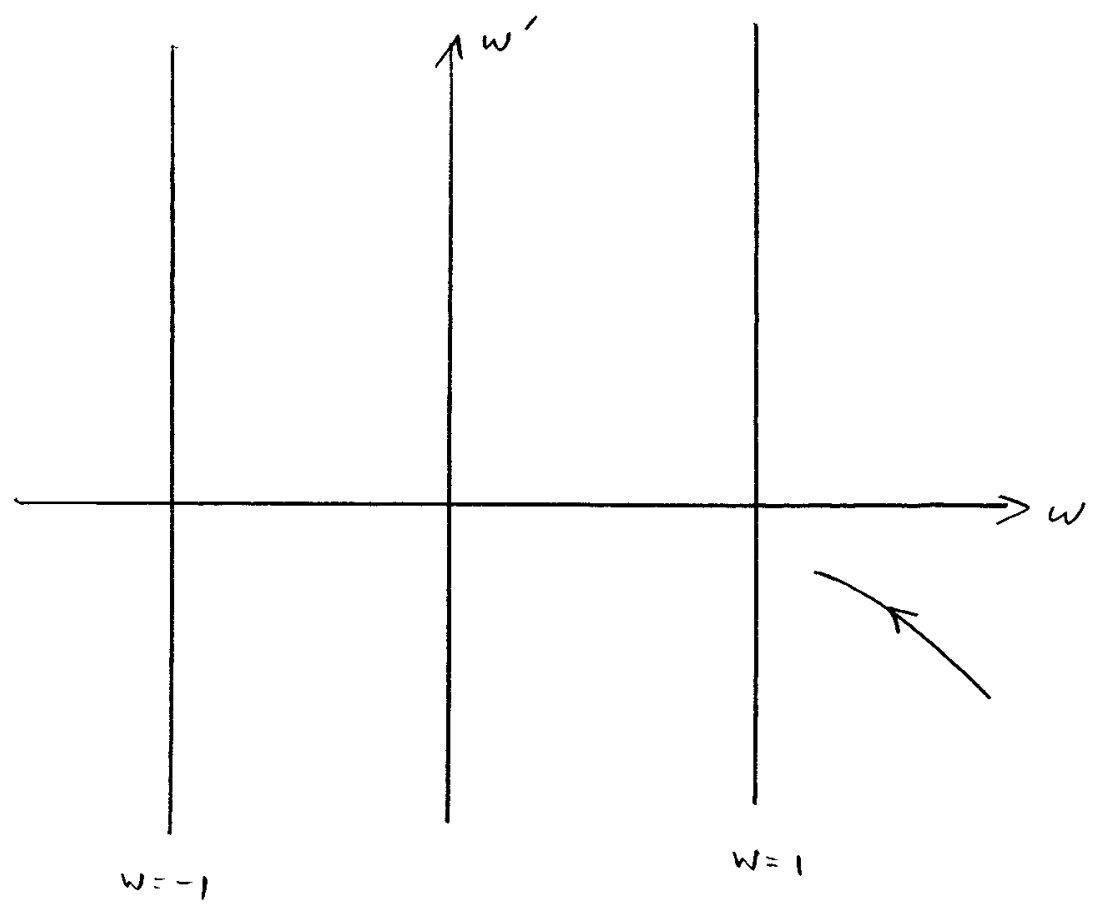

FIG. 4. $w^{\prime}<0$ for $r$ near 0 .

for some constant $c_{1}^{2} \neq 0$. Also,

$$
\frac{\Phi}{r^{2}} w^{\prime 3}+\frac{u w}{r^{2}} w^{\prime 2}=\frac{1}{r} w^{\prime 3}-\frac{A}{r} w^{\prime 3}-\frac{u^{2}}{r^{3}} w^{\prime 3}+\frac{u w}{r^{2}} w^{\prime 2},
$$

and as $\bar{r}>0$, we see that for $r$ near $\bar{r}$,

$$
\left(\frac{\Phi}{r^{2}} w^{\prime 3}+\frac{u w}{r^{2}} w^{\prime 2}\right) \text { is well approximated by }-c_{2}^{2} w^{4} w^{\prime 3}
$$

for some constant $c_{2}^{2} \neq 0$. Thus, for $r$ near $\bar{r}$, solutions of (3.33) are well approximated by the equation

$$
-c_{1}^{2} w^{4} w^{\prime \prime}-c_{2}^{2} w^{4} w^{\prime 3}=0
$$

or, writing $c^{2}=\left(c_{2} / c_{1}\right)^{2}$, (3.36) becomes

$$
w^{\prime \prime}+c^{2} w^{\prime 3}=0
$$

where $c$ is a nonzero constant. Now the solution of (3.37) satisfying (3.32) is

$$
w^{\prime}(r)=-\frac{1}{\sqrt{2 c^{2}}} \frac{1}{\sqrt{r-\bar{r}}}, \quad r>\bar{r},
$$

which implies that $w(r)$ is bounded near $\bar{r}$, contrary to (3.31). This contradiction completes the proof of Proposition 10. 
Proposition 10 yields a few useful corollaries. First, if we define $v(r)$ by (cf. Ref. 8)

$$
v(r)=\left(A w^{\prime}\right)(r),
$$

then $v$ satisfies the equation

$$
v^{\prime}+\frac{2 w^{\prime 2}}{r} v+\frac{u w}{r^{2}}=0
$$

Corollary 11: $\lim _{r \backslash \bar{r}} v(r)=0$.

Proof: $v^{2}(r)=A(r)\left(A w^{\prime 2}(r)\right) \rightarrow 0$ as $r \searrow \bar{r}$.

Corollary 12: $w(r)$ is bounded for $r$ near $\bar{r}$, and if $\bar{r}=0$, then $\lim w^{2}(r)=1$.

Proof: Consider Eq. (2.1): the left side is bounded near $\bar{r}$ so that $u^{2} / r^{2}$ is also bounded near $\bar{r}$. If $\bar{r}>0$ then $u^{2}$ is bounded, so $w$ is bounded near $\bar{r}$, while if $\bar{r}=0$, then $w^{2}(r) \rightarrow 1$ as $r \searrow \bar{r}$.

We shall now consider the case where $w^{\prime}$ is bounded near $\bar{r}$.

Proposition 13: Assume that there is an $M>0$ such that $\left|w^{\prime}(r)\right| \leqslant M$ for $r$ near $\bar{r}$, then Theorem 1 holds.

To prove this proposition, we shall need a lemma.

Lemma 14: If $w^{\prime}$ is bounded near $\bar{r}$, then

$$
\begin{aligned}
& A \text { is of one sign near } \bar{r}, \\
& w^{\prime} \text { is of one sign near } \bar{r},
\end{aligned}
$$

and

$$
\lim _{r \searrow \bar{r}} \frac{u^{2}}{r^{2}}=1 .
$$

Proof: First note that $w^{\prime}$ bounded near $\bar{r}$ implies that $w$ is uniformly continuous near $\bar{r}$, so that $\lim _{r \backslash \bar{r}} w(r)=\bar{w}$ exists. Next, since $w^{\prime}$ is bounded near $\bar{r},(2.1)$ shows that $\lim _{r} \backslash \bar{r}\left(u^{2} / r^{2}\right)=1$, so (3.42) holds and

$$
\frac{u}{r} \rightarrow \pm 1, \quad \text { as } r \searrow \bar{r}
$$

Now writing (3.39) in the form

$$
r^{2} v^{\prime}+2 w^{\prime 2} v r+u w=0
$$

we see that if $\bar{w} \neq 0, \lim _{r \backslash \bar{r}} r v^{\prime}(r)= \pm \bar{w} \neq$, so $v^{\prime}$ is of one sign near $\bar{r}$, and using Corollary 11, $v$ is of one sign near $\bar{r}$, so (3.40) and (3.41) hold. On the other hand, if $\bar{w}=0,(3.42)$ implies that $\bar{r}=1$, so from (3.39), we have $v^{\prime}(1)=0$ and $v^{\prime \prime}(1) \neq 0$. Thus $v$ is again of one sign near $\bar{r}$ so (3.40) and (3.41) hold.

We can now give the following.

Proof of Proposition 13: The last-lemma implies that $A$ is of one sign near $\bar{r}$. If $A>0$ near $\bar{r}$, then the result in Ref. 7 (cf. the remark after the proof of Proposition 2), shows that $(A, w)$ is the ERN solution. Thus we may assume that

$$
A(r)<0 \text {, if } r \text { is near } \bar{r} \text {, }
$$

and we shall show that this leads to a contradiction. 
First, suppose that $\bar{w}=0$. Then from (3.42), we see that $\bar{r}=1$, and as in the proof of Proposition 2, the solution must be the ERN solution. Thus, we may assume that

$$
\bar{w} \neq 0 .
$$

Let $\bar{r}<r_{2}<r_{3}$ where $r_{3}$ is near $\bar{r}$. We consider two cases:

$$
\bar{u} \bar{w} \neq 0
$$

or

$$
\bar{u} \bar{w}=0 .
$$

Using (2.7),

$$
\int_{r_{2}}^{r_{3}} r^{2} A w^{\prime \prime} d r+\int_{r_{2}}^{r^{3}} \Phi w^{\prime} d r+\int_{r_{2}}^{r_{3}} u w d r=0
$$

Suppose first that (3.47) holds. Then

$$
\int_{r_{2}}^{r_{3}} r^{2} A w^{\prime \prime} d r=\left.r^{2} A w^{\prime}\right|_{r_{2}} ^{r_{3}}-\int_{r_{2}}^{r_{3}}\left(r^{2} A\right)^{\prime} w^{\prime} d r,
$$

and letting $r_{2} \searrow \bar{r}$ gives, for some intermediate point $\xi$,

$$
\int_{\bar{r}}^{r_{3}} r^{2} A w^{\prime \prime}=r_{3}^{2} A\left(r_{3}\right) w^{\prime}\left(r_{3}\right)-\left[\xi^{2} A^{\prime}(\xi)+2 \xi A(\xi)\right] w^{\prime}(\xi)\left(r_{3}-\bar{r}\right),
$$

so that

$$
\int_{r_{2}}^{r_{3}} r^{2} A w^{\prime \prime} d r=o\left(r_{3}-\bar{r}\right)
$$

Similarly, since $\Phi(r)=r-r A-u^{2} / r \rightarrow 0$ as $r \searrow \bar{r}$ [in view of (3.42)], we have, for some intermediate point $\eta$,

$$
\lim _{r_{2} \searrow \bar{r}} \int_{r_{2}}^{r_{3}} \Phi w^{\prime} d r=\int_{\bar{r}}^{r_{3}} \Phi w^{\prime} d r=\left(\Phi w^{\prime}\right)(\eta)\left(r_{3}-\bar{r}\right)=o\left(r_{3}-\bar{r}\right)
$$

Finally, we have, for some intermediate point $\zeta$,

$$
\lim _{r_{2} \searrow \bar{r}} \int_{r_{2}}^{r_{3}} u w d r=\int_{\bar{r}}^{r_{3}} u w d r=(u w)(\eta)\left(r_{3}-\bar{r}\right)=O\left(r_{3}-\bar{r}\right)
$$

where the constant is nonzero, in view of (3.47). Taking the limit $r_{2} \searrow \bar{r}$ in (3.49), and using (3.50)-(3.51) gives the contradiction

$$
o\left(r_{3}-\bar{r}\right)=O\left(r_{3}-\bar{r}\right) .
$$

Now suppose that (3.48) holds. In view of (3.46), this means $\bar{u}=0$ so (3.42) implies $\bar{r}=0$. Thus, $\bar{w}= \pm 1$, and, for definiteness, suppose that $\bar{w}=1$ (the proof for $\bar{w}=-1$ is similar, and will be omitted). As above, (3.49) gives 


$$
\int_{0}^{r_{3}} r^{2} A w^{\prime \prime} d r=r_{3}^{2} A\left(r_{3}\right) w^{\prime}\left(r_{3}\right)-\int_{0}^{r_{3}}\left(r^{2} A^{\prime}(r)+2 r A(r)\right) w^{\prime}(r) d r .
$$

But

$$
r_{3}^{2} A^{\prime}\left(r_{3}\right) w^{\prime}\left(r_{3}\right)=o\left(r_{3}^{3}\right)
$$

[since $A\left(r_{3}\right)=o\left(r_{3}\right)$, and for some intermediate point $\xi$,

$$
\int_{0}^{r_{3}}\left(r^{2} A^{\prime}+2 r A(r)\right) w^{\prime}(r) d r=\left(\xi^{2} A^{\prime}(\xi)+2 \xi A(\xi)\right)\left(w\left(r_{3}\right)-w(0)\right)=o\left(r_{3}^{3}\right) ;
$$

thus

$$
\int_{0}^{r_{3}} r^{2} A w^{\prime \prime} d r=o\left(r_{3}^{3}\right)
$$

Similarly, for some intermediate point $\eta$,

$$
\int_{0}^{r_{3}} \Phi w^{\prime} d r=\left(\Phi w^{\prime}\right)(\eta) r_{3}=o\left(r_{3}^{3}\right),
$$

because

$$
\Phi(r)=r\left(1-\frac{u^{2}}{r^{2}}-A\right)=r\left(r A^{\prime}+2 A w^{\prime 2}\right)=o\left(r_{3}^{2}\right) .
$$

However, for some intermediate point $\zeta$, we have

$$
\int_{0}^{r_{3}} u w d r=\int_{0}^{r_{3}} \frac{u}{r} w r d r=\frac{u(\zeta)}{\zeta} w(\zeta) \frac{r_{3}^{2}}{2}=O\left(r_{3}^{2}\right)
$$

and this gives a contradiction, in view of (3.53) and (3.54). This completes the proof of Proposition 13.

In view of this last result, we may assume in what follows that

$$
w^{\prime}(r) \text { is unbounded for } r \text { near } \bar{r} \text {. }
$$

Our strategy for completing the proof of Theorem 1 is to first show that Theorem 1 holds, provided that both $A(r)$ and $w^{\prime}(r)$ are of one sign (not necessarily the same), for $r$ near $\bar{r}$, and then to prove that this assumption is always valid.

Proposition 15: Assume that $w^{\prime}(r)$ and $A(r)$ each are of one sign for $r$ near $\bar{r}$; then Theorem 1 holds.

Proof: As we have remarked earlier, we may assume that (3.45) holds, and also in view of Proposition 13, we may also assume that (3.55) holds. We shall show that our assumptions lead to a contradiction.

First recall that since $A w^{\prime 2}$ is bounded near $\bar{r}$, it follows from Corollary 12 that for $r$ near $\bar{r}$, $w$ is bounded, and if $\bar{r}=0$, then $w^{2}(r) \rightarrow 1$. Furthermore, as $w^{\prime}$ is of one sign near $\bar{r}, \bar{w}$ $=\lim _{r \backslash \bar{r}} w(r)$ exists and is finite. We assume for definiteness that

$$
w^{\prime}(r)>0, \text { for } r \text { near } \bar{r}
$$

if $w^{\prime}<0$ near $\bar{r}$, the proof is similar. 


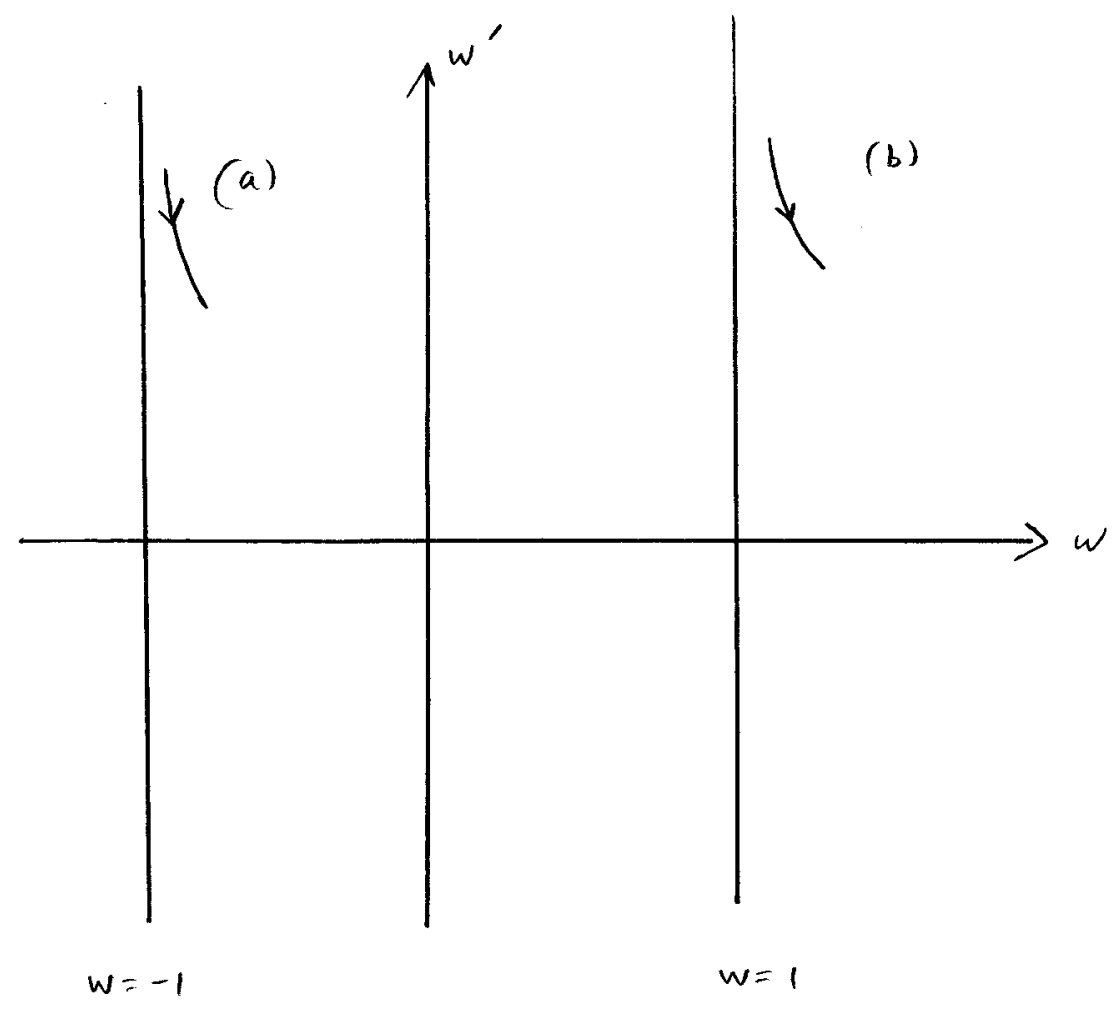

FIG. 5. $w^{\prime}>0, w^{2}$ near 1 , for $r$ near $\bar{r}$.

The proof is now divided into two cases: $\bar{r}=0$ and $\bar{r}>0$.

Case 1. $\bar{r}=0$. Since $\lim _{r \backslash 0} w^{2}(r)=1$, (3.56) implies that for $r$ near 0 , either (a) $-1<w(r)$ $<0$, or (b) $1<w(r)<1+\epsilon$, for some small $\epsilon>0$; cf. Fig. 5. Now from Corollary 11, $v(0)=0$, and from (3.19), $v^{\prime}(r)>0$, for $r$ near 0 , and $v^{\prime}(0) \geqslant 0$. This implies that $v(r)>0$ for $r$ near 0 , and this is impossible because $A<0$ and $w^{\prime}>0$.

Case 2. $\bar{r}>0$. Since $w$ has a finite limit at $\bar{r}$, it follows from $(2.1)$ that $\lim _{r \backslash 0}\left(A w^{\prime 2}\right)(r)$ $=-L$, where $L \geqslant 0$. If $L>0$, then $w^{\prime 2} \rightarrow \infty$ as $r \backslash \bar{r}$, and (3.56), together with (3.39) shows that $v^{\prime}(\bar{r})>0$, for $r$ near $\bar{r}$, which is impossible, as we have just seen. Thus we may assume that $L=0$.

If $-1 \leqslant \bar{w} \leqslant 0$, or $\bar{w} \geqslant 1$, then (3.19) and (3.39) show that $v^{\prime}(r)>0$ for $r$ near $\bar{r}$ so that if $\epsilon>0$ is small, we have, for some intermediate point $\xi$,

$$
v(\bar{r}+\epsilon)=v(\bar{r}+\epsilon)-v(\bar{r})=\epsilon v^{\prime}(\xi)>0,
$$

and this is a contradiction. Thus, we can assume that $\bar{w}<-1$, or $0<\bar{w}<1$. Now as $L=0,(2.6)$ implies that $\Phi(\bar{r})=0$, so $\bar{u}=\bar{r}$. Also, from Refs. 8 and 9 ,

$$
\Phi^{\prime}(\bar{r})=\frac{2 u^{2}}{\bar{r}^{2}}+2 A w^{\prime 2}+\frac{4 u w w^{\prime}}{\bar{r}}
$$

so that $\Phi^{\prime}(\bar{r})>0$. Thus $\Phi(r)>0$ for $r>\bar{r}, r$ near $\bar{r}$, so (2.6) implies that $A^{\prime}(r)>0$ for $r$ near $\bar{r}$, and hence as $A(\bar{r})=0$, we get the contradiction $A(r)>0$ for $r$ near $\bar{r}$. This completes the proof of Proposition 15.

Now in view of Corollary 5, we may assume that for $r$ near $\bar{r}$, one of the following must hold: 


$$
\begin{array}{ll}
\text { (I) } & w^{2}(r)<1, \\
\text { (II) } & w(r)>1, \\
\text { (III) } & w(r)<-1 .
\end{array}
$$

Our objective is to show that in each of these cases, both $A(r)$ and $w^{\prime}(r)$ have fixed signs for $r$ near $\bar{r}$. Then Proposition 15 will complete the proof of Theorem 1. We begin with the most difficult case; namely the following.

Case I: $w^{2}(r)<1$ for $r$ near $\bar{r}$.

In order to carry out our program in this case, we shall first rule out "infinite rotation" of the orbit $\left(w(r), w^{\prime}(r)\right)$ about the origin. There are two cases to consider; namely

$$
\lim _{r} w(r)=0, \quad \text { and } w^{\prime} \text { is not of one sign near } \bar{r},
$$

or

$$
-1 \leqslant \overline{\lim }_{r \searrow r} w(r) \leqslant 0 \leqslant \varlimsup_{r \searrow \bar{r}} w(r) \leqslant 1
$$

and

$$
\lim _{r \searrow r} w(r)<\varlimsup_{r \searrow \bar{r}} w(r)
$$

cf. Figs. 6 and 7.

Proposition 16: It is impossible for (3.57) to hold.

Proof: Define $Q^{\prime}(r)=2 w^{\prime 2} / r, Q\left(r_{1}\right)=0$. Then, $Q(r)<0$ if $\bar{r} \leqslant r<r_{1}$ and (2.1) can be written as

$$
\left(r e^{Q} A\right)^{\prime}=\left(1-\frac{u^{2}}{r^{2}}\right) e^{Q}
$$

Also, $\left|r e^{Q(r)} A(r)\right| \leqslant|r A(r)|$, so that

$$
r e^{Q(r)} A(r) \rightarrow 0, \quad \text { as } r \searrow \bar{r} .
$$

Now if $\bar{r}-1 / \bar{r} \geqslant 0$, then (3.57) and (3.59) imply that for $r$ near $\bar{r},\left(r e^{Q} A\right)^{\prime} \geqslant 0$, so that for such $r$,

$$
r e^{Q(r)} A(r)=r e^{Q(r)} A(r)-\bar{r} e^{Q(\bar{r})} A(\bar{r})=\left(\xi e^{Q(\xi)} A(\xi)\right)^{\prime}>0,
$$

for some intermediate point $\xi$. It follows that $A(r)>0$ for $r$ near $\bar{r}$, and this is contrary to our assumptions; cf. the remark after the proof of Proposition 2.

If $\bar{r}-1 / r<0$, then a similar argument shows that $A(r)<0$ for $r$ near $\bar{r}$. Thus from (2.7), if $w^{\prime}(r)=0$, then $w^{\prime \prime}(r)>0$, for $0<w(r)<1$, and $w^{\prime \prime}(r)<0$, if $-1<w(r)<0$; cf. Fig. 8. Thus, $w^{\prime}(r)$ is of one sign for $r$ near $\bar{r}$, contrary to our assumption (3.57). This completes the proof of Proposition 16.

We now turn to the remaining case; namely (3.58). To handle, this case, we first note that

$$
\bar{r}>0,
$$

if (3.58) holds. Indeed, (3.58) implies that we can find a sequence $r_{n} \searrow \bar{r}$ such that $w\left(r_{n}\right)=0$. Then (2.1) gives 


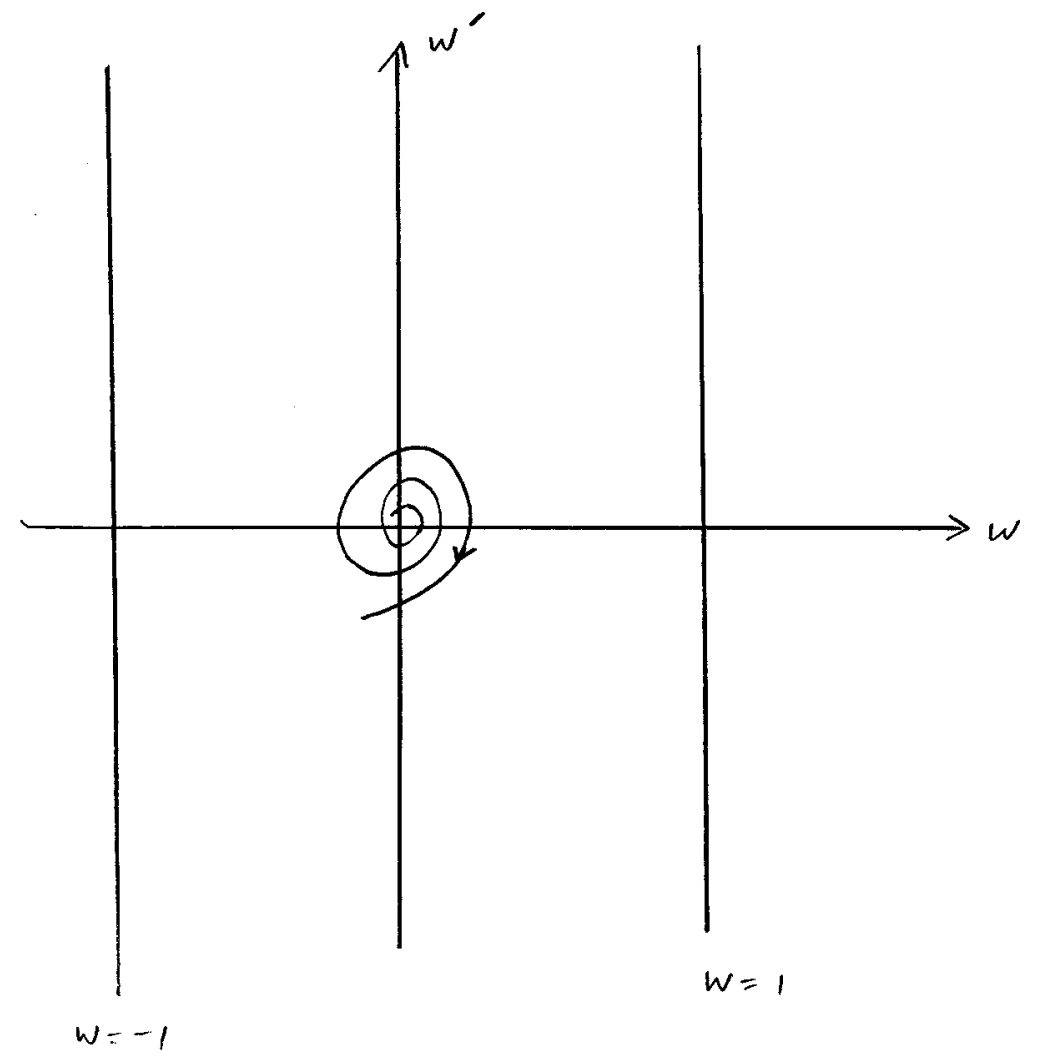

FIG. 6. The case where (3.57) holds.

$$
r_{n} A^{\prime}\left(r_{n}\right)+\left(1+2 w^{\prime 2}\left(r_{n}\right)\right) A\left(r_{n}\right)=1-\frac{1}{r_{n}^{2}},
$$

and since the left side is bounded (Proposition 10), it follows that $\left\{1 / r_{n}^{2}\right\}$ is also bounded, and this proves (3.61).

Proposition 17: It is impossible for (3.58) to hold.

Proof: If (3.58) holds, then we can find an $\epsilon$,

$$
0<\epsilon<\frac{1}{4}
$$

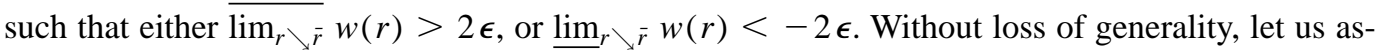
sume that the former inequality holds; cf. Fig. 9.

Thus there exist sequences of points $\left\{a_{n}\right\}$ and $\left\{b_{n}\right\}$, such that

$$
\begin{gathered}
b_{n}>a_{n}>b_{n+1}>\bar{r}, \quad b_{n} \rightarrow \bar{r}, \\
w\left(a_{n}\right)=0, \quad w\left(b_{n}\right)=2 \epsilon,
\end{gathered}
$$

and if $a_{n}<r<b_{n}$, then

$$
w\left(a_{n}\right)<w(r)<w\left(b_{n}\right),
$$




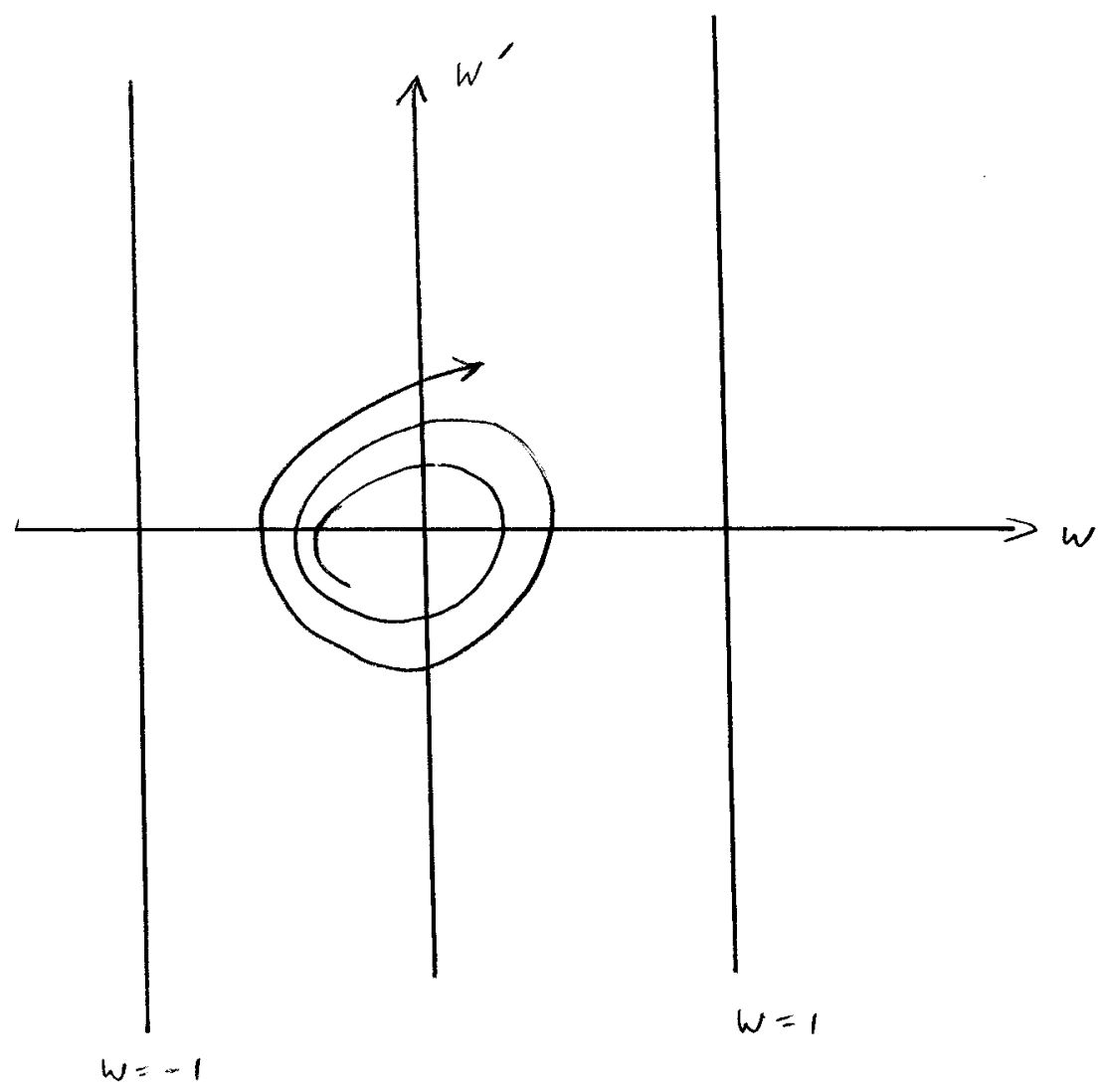

FIG. 7. The case where (3.58) holds.

and finally,

$$
w^{\prime}(r)>0
$$

see Fig. 9. We note that the intervals $\left[a_{n}, b_{n}\right]$ are all disjoint from each other.

Lemma 18: There exist $\delta>0, \eta>0$, and an integer $N>0$, and sequences $\left\{c_{n}\right\},\left\{d_{n}\right\}$, defined for $n \geqslant N$,

$$
a_{n} \leqslant c_{n}<d_{n} \leqslant b_{n},
$$

such that if $r$ satisfies $c_{n} \leqslant r \leqslant d_{n}$, then

$$
\begin{gathered}
A(r) \quad \text { is of one sign, } \\
|\phi(r)| \geqslant \eta, \\
w\left(d_{n}\right)-w\left(c_{n}\right) \geqslant \delta .
\end{gathered}
$$

Before giving the proof, we shall need a few lemmas. We begin with the following easy result. Lemma 19: Suppose $\Phi(A(r), w(r), r) \equiv \Phi(r) \neq 0$ on $I=[a, b]$, where $a>0$. Then $A$ can have at most one zero on $I$. 


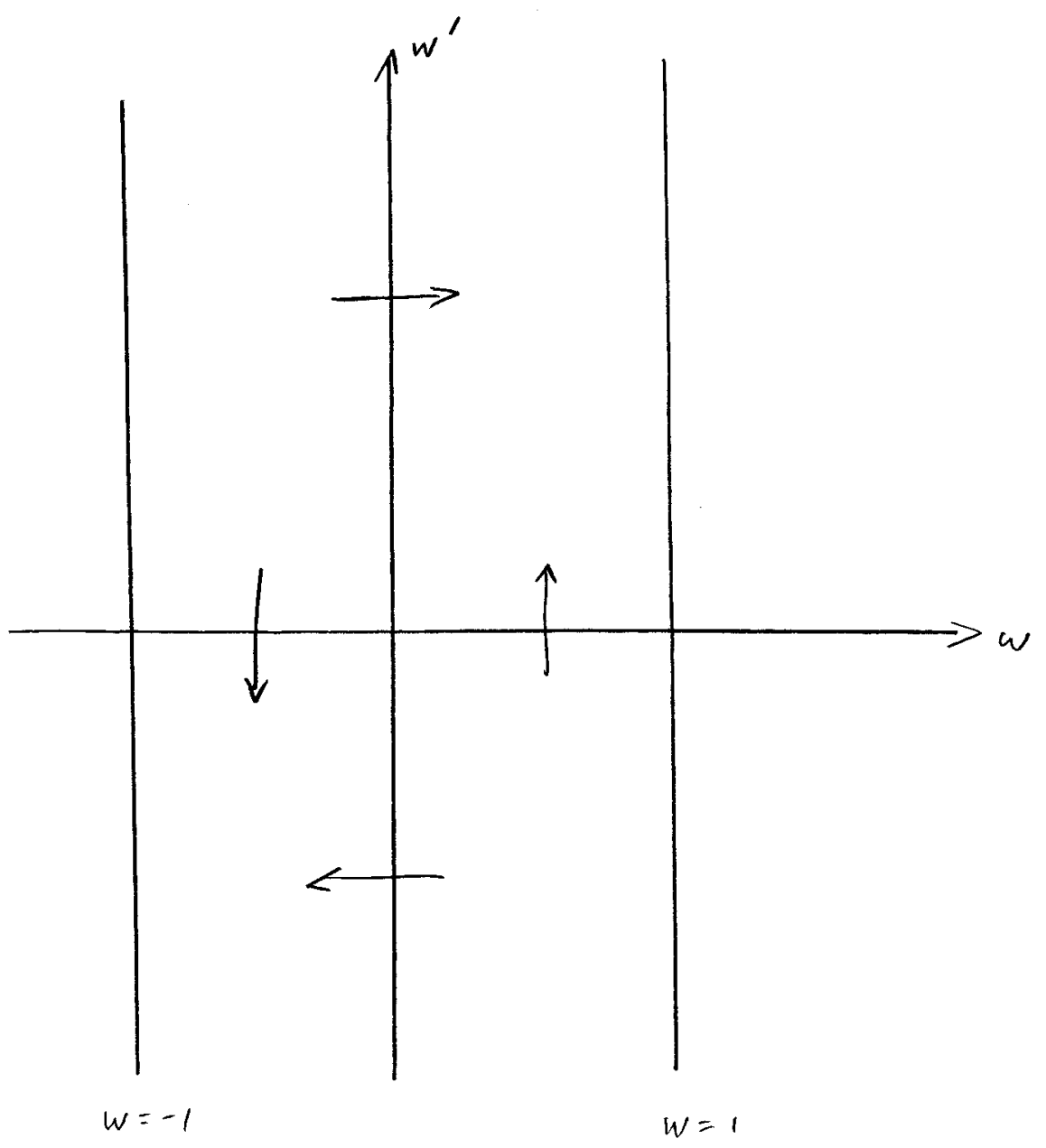

FIG. 8. $w^{\prime}$ is of one sign near $\bar{r}$.

Proof: From (2.6), if $A(r)=0$ for some $r \in I$, we see $r A^{\prime}(r)=\Phi(r) / r \neq 0$. Thus $A^{\prime}$ is of one sign, and this implies that $A$ can have at most one zero on $I$.

Lemma 20: Suppose $\Phi(A(r), w(r), r) \equiv \Phi(r) \neq 0$ on the interval $I=[\mathrm{a}, \mathrm{b}]$, where $a>0$, and that $w(a)=0, w(b)=2 \epsilon>0$. Then there exist numbers $c, d, a \leqslant c<d \leqslant b$ such that $w(d)-w(c) \geqslant \epsilon / 2$, and $A$ has one sign on $[c, d]$.

Proof: We break the proof up into two cases; namely, $A(a) A(b) \neq 0$ and $A(a) A(b)=0$.

Case 1. $A(a) A(b) \neq 0$.

If $\operatorname{sgn} A(a)=\operatorname{sgn} A(b)$, then set $c=a, d=b$, and the result holds in view of Lemma 19. If $\operatorname{sgn} A(a) \neq \operatorname{sgn} A(b)$, choose $z$ such that $w(z)=\epsilon, a<z<b$. If $A(z) \neq 0$, then in view of Lemma 19 , one of the following must hold; namely, either $A(a) A(z)>0$ or $A(z) A(b)>0$. In the first case, choose $c=a, d=z$, and in the second case, choose $c=z, d=b$. If $A(z)=0$, choose $z^{\prime} \in I$ with $w\left(z^{\prime}\right)=3 \epsilon / 2$. Then $A\left(z^{\prime}\right) \neq 0$, and we proceed as before to reach the desired conclusion; this completes the proof in case 1 .

Case 2. $A(a) A(b)=0$.

In view of Lemma 19, we cannot have both $A(a)=0$ and $A(b)=0$. Thus suppose $A(a)=0$, $A(b) \neq 0$; (the proof in the other case is similar). Now choose $z \in I, z$ near $a$, such that $A(z) \neq 0$, 


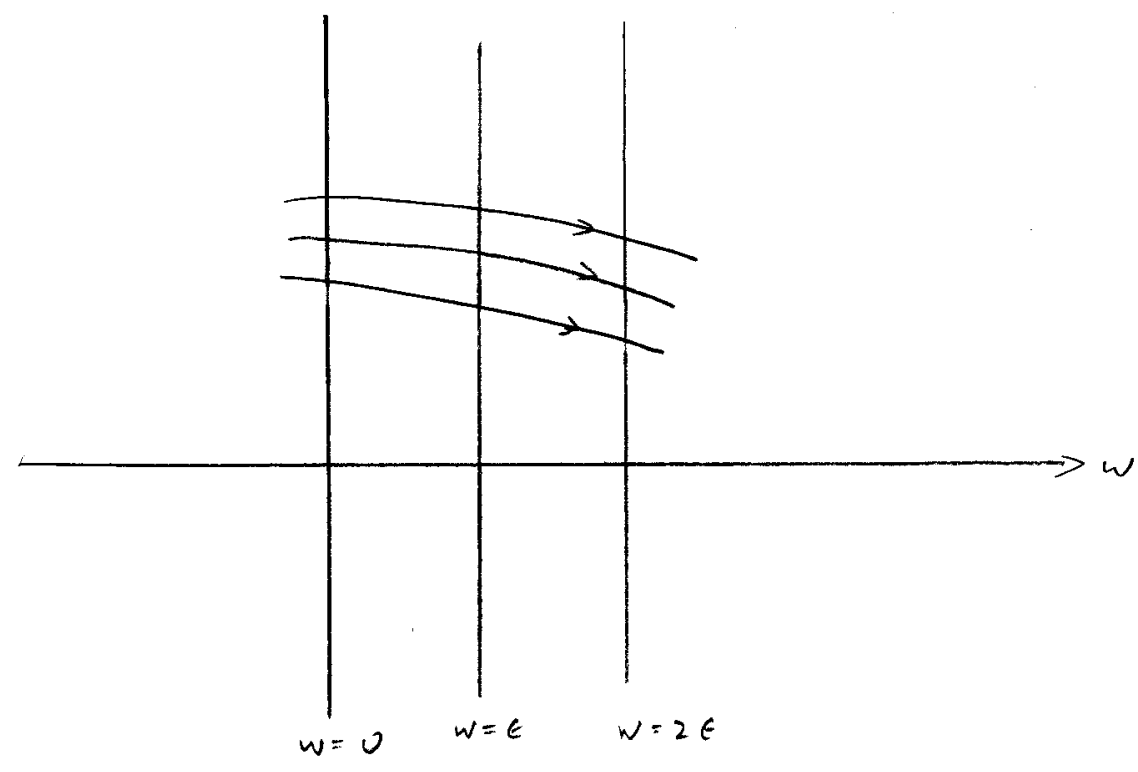

FIG. 9. $\varlimsup_{r \backslash, \bar{r}} w(r)>2 \epsilon$.

and apply the result in Case 1 to the situation where $a$ is replaced by $z$. This proves Lemma 20 .

Proof of Lemma 18: We break the proof up into three cases: $\bar{r}>1, \bar{r}<1, \bar{r}=1$.

Suppose first that $\bar{r}>1$. Then set

$$
\bar{r}-\frac{1}{\bar{r}}=2 \eta,
$$

where $\eta>0$. Since $\lim _{r \backslash \bar{r}} A(r)=0$, then if $r>\bar{r}$,

$$
\Phi(A, w, r)=r-r A-\frac{u^{2}}{r}>r-r A-\frac{1}{r}>\bar{r}-\frac{1}{\bar{r}}-r A .
$$

Thus there exists a $\sigma, 0<\sigma<\epsilon$ such that if $0<r-\bar{r}<\sigma$, then

$$
\Phi(A, w, r)>\eta .
$$

Now we only consider those $n$ for which $b_{n}<\bar{r}+\sigma$; [cf. (3.62)]. Then with $I_{n}=\left[a_{n}, b_{n}\right]$, we apply Lemma 20 to conclude that (3.65) $-(3.68)$ hold, with $\delta=\epsilon / 2$.

Suppose now that $\bar{r}<1$. Then set

$$
\bar{r}-\frac{1}{\bar{r}}=-2 \eta,
$$

where $\eta>0$. Since

$$
\Phi(A, w, r)=r-\frac{u^{2}}{r}-r A,
$$

and $r A(r) \rightarrow 0$ as $r \backslash \bar{r}$, and $u(r)=1-w^{2}(r)$, we see that we can find a $\sigma>0$ such that if $0<r$ $-\bar{r}<\sigma$, and $0<w<\sigma$, 


$$
\Phi(A, w, r)<-\eta .
$$

Again, we only consider those $n$ for which $b_{n}<\bar{r}+\sigma$. Now as $0 \leqslant w(r) \leqslant 2 \epsilon$ if $a_{n} \leqslant r \leqslant b_{n}$, we choose $b_{n}^{\prime}$ such that $w\left(b_{n}^{\prime}\right)=\min (\sigma, 2 \epsilon)$. Then $\Phi(r)<-\eta$ on the interval $\left[a_{n}, b_{n}^{\prime}\right]$. Another application of Lemma 20 shows that we can achieve (3.65)-(3.68).

Finally, consider the case $\bar{r}=1$. Consider $w$ satisfying $\epsilon \leqslant w \leqslant 2 \epsilon$. Then for these $w$,

$$
1-\frac{u^{2}}{r} \geqslant 1-\left(1-\epsilon^{2}\right)^{2} \equiv 2 \eta>0,
$$

since $\epsilon<1 / 4$. Thus, if $r$ is sufficiently close to 1 , say $0<r-1<\sigma$, then $\Phi(A, w, r)>\eta$. Taking $n$ so large that $b_{n}<\bar{r}+\sigma$, and defining $c_{n}$ by $w\left(c_{n}\right)=\epsilon$, and setting $d_{n}=b_{n}$, we see that Lemma 20 again applies, and we can achieve (3.65)-(3.68). This completes the proof of Lemma 18.

We now can give the following proof.

Proof of Proposition 17: From Lemma 18, we see that we have infinitely many intervals, $J_{n}=\left[c_{n}, d_{n}\right] \subset\left[a_{n}, b_{n}\right]$, where (3.66)-(3.68) hold on $J_{n}$. We now consider two cases.

Case $a$. For infinitely many intervals $J_{n}$,

$$
(A \Phi)(r)>0, \quad \text { if } r \in J_{n} .
$$

Case $b$. For all but a finite number of intervals $J_{n}$,

$$
(A \Phi)(r)<0 \quad \text { if } r \in J_{n} .
$$

The proof is somewhat involved, so before giving the details, we shall discuss the strategy. The basic idea is to show that there is a $\zeta>0$ such that

$$
d_{n}-c_{n} \geqslant \zeta
$$

then since $\bar{r}<a_{n} \leqslant c_{n}<d_{n} \leqslant b_{n}$ and $b_{n} \rightarrow \bar{r}$, (3.73) would give the desired contradiction. Now, in order to carry out this program, we need the following fundamental lemma.

Lemma 21. Let $0<\bar{r}<\alpha<\beta<2 \bar{r}$, and assume that $(A, w)$ is a solution of (2.1), (2.2) on $J_{+}=[\alpha, \beta]$ or $J_{-}=[-\beta,-\alpha]$. Assume too that on this interval, $w^{2}(r)<1$,

$$
0<\left|\left(A w^{\prime 2}\right)(\bar{r})\right|<L
$$

and

$$
|\Phi(r)| \geqslant \eta,
$$

for some positive constants $L$ and $\eta$. Then

$$
|\beta-\alpha| \geqslant \zeta>0,
$$

where $\zeta$ is a constant depending only on $\bar{r}, L, \eta$, and

$$
\Delta \equiv|w(\beta)-w(\alpha)| .
$$

Proof: Assume first that we are on $J_{+}$, and that on $J_{+}$both

$$
w^{\prime}>0
$$

and

$$
A \Phi>0 .
$$


Define the constant $m$ by

$$
m=\max \left(\frac{1}{\eta}, \frac{16 \bar{r}^{2} L}{\eta \Delta}\right)
$$

We claim that

$$
\text { if } r \in J_{+} \quad \text { and } \quad w^{\prime}(r) \geqslant m \text {, then } \quad w^{\prime \prime}(r)<0 .
$$

To see this, we have from (2.7),

$$
r^{2} A w^{\prime \prime}+\Phi w^{\prime}+u w=0,
$$

and as $\left|\Phi w^{\prime}\right|>1$ and $|u w|<1 / 2$ on $J_{+}$, it follows that $\operatorname{sgn}\left(\Phi w^{\prime}+u w\right)=\operatorname{sgn} \Phi w^{\prime}=\operatorname{sgn} \Phi$; thus $\operatorname{sgn} w^{\prime \prime}=\operatorname{sgn}(-A \Phi)<0$, and this proves (3.85). It follows from this that

$$
\text { if } \tilde{r} \in J_{+} \quad \text { and } \quad w^{\prime}(\tilde{r}) \leqslant m, \quad \text { then } w^{\prime}(r)<m \text { for } r>\tilde{r}, \quad r \in J_{+} .
$$

Now define $\xi$ by

$$
w(\xi)=\frac{w(\alpha)+w(\beta)}{2} .
$$

Then if for some $\tilde{r} \in J_{+}, \tilde{r} \leqslant \xi$, we have $w^{\prime}(\tilde{r}) \leqslant m$, then $w^{\prime}(r) \leqslant m$ for all $r \geqslant \tilde{r}$. In this case, we would have, for some intermediate point $\theta$,

$$
|\beta-\alpha| \geqslant|\beta-\tilde{r}|=\frac{\mid w(\beta)-w)(\tilde{r}) \mid}{\left|w^{\prime}(\theta)\right|} \geqslant \frac{\Delta / 2}{m}=\zeta,
$$

and this would prove the result. Thus, we may assume that

$$
w^{\prime}(r) \geqslant m, \quad \text { for all } r \in J_{+}, \quad r \leqslant \xi .
$$

We will show that this leads to a contradiction.

We first claim that

$$
\frac{w^{\prime \prime}}{w^{\prime 2}}<\frac{-\Phi w^{\prime}}{2 r^{2} A w^{\prime 2}}, \quad \text { if } \quad r \in J_{+}
$$

Indeed, (2.7) gives

$$
\frac{-w^{\prime \prime}}{w^{\prime 2}}=\frac{\Phi w^{\prime}+u w}{r^{2} A w^{\prime 2}}>\frac{\Phi w^{\prime}}{2 r^{2} A w^{\prime 2}}
$$

because $\left|\Phi w^{\prime}\right|>1$, and $|u w|<\frac{1}{2}$ imply that $\operatorname{sgn}\left(\Phi w^{\prime}+u w\right)=\operatorname{sgn}\left(\Phi w^{\prime}\right)$. Thus using (3.78), $\operatorname{sgn}\left[\left(\Phi w^{\prime}+u w\right) / r^{2} A w^{\prime 2}\right]=\operatorname{sgn}\left[\Phi w^{\prime} / A\right]=\operatorname{sgn} w^{\prime}>0$, and this gives (3.84).

Next, we show

$$
\frac{-\Phi w^{\prime}}{2 r^{2} A w^{\prime 2}} \leqslant-c^{2} w^{\prime}(r), \quad \text { if } r \in J_{+}, \quad r \leqslant \xi
$$

where 


$$
c^{2}=\frac{\eta}{8 \bar{r}^{2} L} .
$$

To see this we have, for $r \in J_{+}, r \leqslant \xi$,

$$
\frac{\Phi w^{\prime}}{2 r^{2} A w^{\prime 2}}=\left|\frac{\Phi w^{\prime}}{2 r^{2} A w^{\prime 2}}\right| \geqslant \frac{\eta w^{\prime}}{2 r^{2} L} \geqslant \frac{\eta w^{\prime}}{8 \bar{r}^{2} L}
$$

since $r \leqslant \xi<\beta \leqslant 2 \bar{r}$, and this gives (3.86).

Now if $r \in J_{+}, r \leqslant \xi$, (3.84) and (3.88) give

$$
\frac{w^{\prime \prime}}{w^{\prime 2}} \leqslant-c^{2} w^{\prime}
$$

Integrating this from $\alpha$ to $\xi$ gives

$$
-\frac{1}{w^{\prime}(\xi)}<\frac{1}{w^{\prime}(\alpha)}-\frac{1}{w^{\prime}(\xi)} \leqslant-c^{2}(w(\xi)-w(\alpha))=-\frac{c^{2} \Delta}{2}
$$

so that $w^{\prime}(\xi)<2 / c^{2} \Delta=16 \bar{r}^{2} L / \eta \Delta \leqslant m$, and this contradicts (3.83). Thus the lemma is proved if we are on $J_{+}$, and both (3.78) and (3.79) hold.

Now suppose that we are on $J_{-}$, and both (3.78), and (3.79) hold. It is clear that in this case the same proof works; we merely substitute $-\beta$ for $\alpha$ and $-\alpha$ for $\beta$.

Next, consider the case where $w^{\prime}<0$, (on $J_{+}$or $\left.J_{-}\right)$, and (3.79) holds. Note that if $(A, w)$ is a solution of $(2.1),(2.2)$, then so is $(A,-w)$. Also, if $(A, w)$ satisfies the hypotheses of the lemma, so does $(A,-w)$; this shows that the case $w^{\prime}<0$ is reduced to the case $w^{\prime}>0$.

Finally, suppose that $A \phi<0$, on $J$ (where $J=J_{+}$or $J_{-}$). We extend the functions $A$ and $w$ to $-J$ by defining

$$
(A(-r), w(-r))=(A(r), w(r)), \quad r \in J .
$$

Since $\Phi(-r)=-\Phi(r)$, we see that $A \Phi>0$ on $-J$. Thus, applying what we have already proved to $-J$ gives that $(-A,-w)$ satisfies the conclusions of the lemma, and hence so does $(A, w)$. This completes the proof of Lemma 21.

We now return to the proof of Proposition 17. For this, we shall use Lemmas 18 and 21. Thus, choose $n$ so large that $b_{n}<2 \bar{r}$; cf. (3.62). Now we apply Lemma 21 to the intervals $J_{n}=\left[c_{n}, d_{n}\right]$; the hypotheses of Lemma 21 are valid because of Proposition 10, and (3.65)-(3.67), of Lemma 18. We conclude that for large $n, d_{n}-c_{n} \geqslant \zeta$, where $\zeta$ is independent of $n$. Thus for large $n$, $b_{n}-a_{n} \geqslant \zeta$, and this contradicts (3.62). The proof of Proposition 17 is complete.

We now return to the proof of Theorem 1. For this, recall that from Corollary 5, we have that for $r$ near $\bar{r}$, one of the following must hold: either $w^{2}(r)<1$, or $w(r)>1$, or $w(r)<-1$. In view of Propositions 16 and 17, the orbit $\left(w(r), w^{\prime}(r)\right)$ cannot have infinite rotation about $(0,0)$; thus for $r$ near $r$, we may assume that the orbit does not cross $w=0$. We shall show that in all of the above cases both $A$ and $w^{\prime}$ are each of fixed sign for $r$ near $\bar{r}$, and then Proposition 15 will complete the proof of Theorem 1.

To carry out this program, we first note that for $r$ near $\bar{r}$, we cannot have both $A(r)=0=w^{\prime}(r)$. Indeed, for $r$ near $\bar{r}$, our above remarks imply that $(u w)(r) \neq 0$, so that $(2.2)$ implies that not both $A(r)$ and $w^{\prime}(r)$ can be zero. Similarly, not both $A(r)$ and $A^{\prime}(r)$ can be zero for some $r>\bar{r}$, since if this were so, (2.6) implies $\Phi(r)=0$ so (2.7) would give the contradiction $(u w)(r)=0$. 


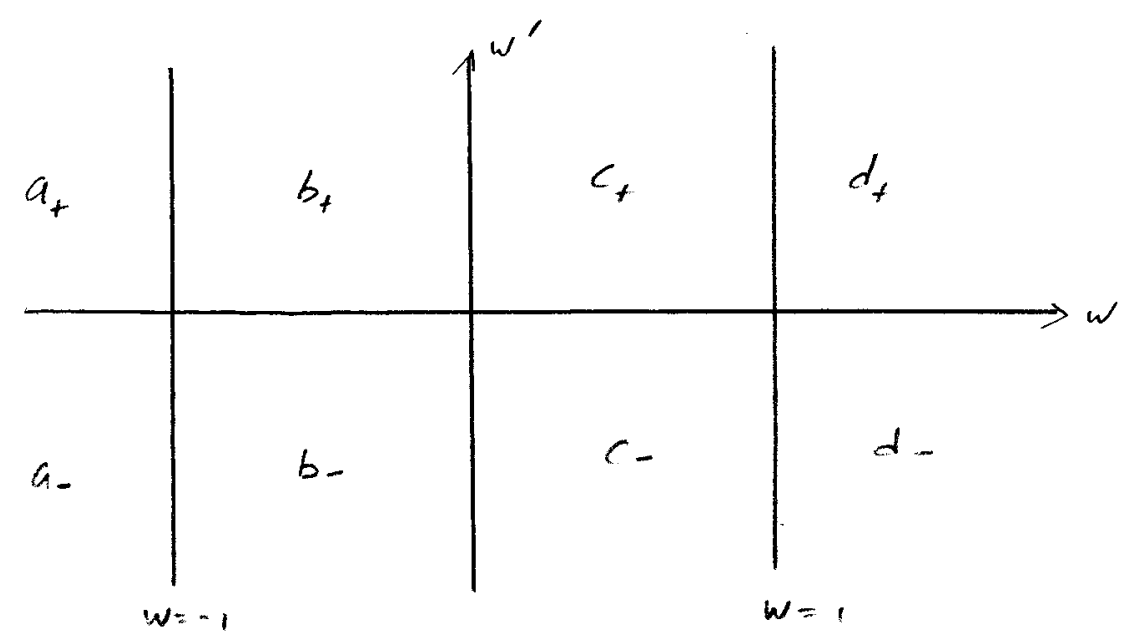

FIG. 10. $a \cup b \cup c \cup d$.

Now let us denote the region $w<-1$ by $a=a_{+} \cup a_{-}$(cf. Fig. 10); the region $-1<w<0$ by $b=b_{+} \cup b_{-}$; the region $0<w<1$ by $c_{+} \cup c_{-}$; and the region $1<w$ by $d_{+} \cup d_{-}$. For $r$ near $\bar{r}$, we may assume that the orbit lies in precisely one of the regions $a, b, c$, or $d$.

Lemma 22: If $v(s)=0$, for $s$ near $\bar{r}$, then $\left(u w v^{\prime}\right)(s)<0$.

Proof: If $s$ is near $\bar{r}$, then $(u w)(s) \neq 0$, by our above remarks. Thus from (3.39),

$$
\left(u w v^{\prime}\right)(s)=-\frac{u^{2}(s) w^{2}(s)}{s^{2}}<0,
$$

and this proves the lemma.

We can now complete the proof of Theorem 1. Namely, if $r$ is near $\bar{r}$, then we have $(u w)(r) \neq 0$. Thus the last lemma shows that $v$ changes sign at every zero. Since $v$ can have at most one sign change in each of the regions $a, b, c$, or $d, A$, and $w^{\prime}$ can have at most one sign change between them. Thus for $r$ near $\bar{r}, A$ and $w^{\prime}$ each are of fixed sign. As we have noted above, Proposition 15 completes the proof of Theorem 1.

\section{ACKNOWLEDGMENTS}

We wish to thank Piotr Bizon and Robert Wald, for explaining the physics of black holes to us.

Both authors are supported in part by National Science Foundation, Grant No. G-DMS 9501128.

\footnotetext{
${ }^{1}$ R. M. Wald, General Relativity (University of Chicago Press, Chicago, 1984).

${ }^{2}$ P. A. Anderson, W. A. Hiscock, and D. J. Lorenz, Phys. Rev. Lett. 74, 4365 (1995).

${ }^{3}$ S. W. Hawking, G. T. Horowitz, and S. F. Ross, Phys. Rev. D 51, 4302 (1995).

${ }^{4}$ J. A. Harvey and A. Strominger, in Recent Directions in Particle Theory-From Superstrings and Black Holes to the Standard Model, edited by J. Harvey and J. Pochinski (World Scientific, Singapore, 1993).

${ }^{5}$ C. F. E. Holzey and F. Wilczek, Nucl. Phys. B 380, 447 (1992).

${ }^{6}$ M. J. Duff and J. Rahmfeld, Phys. Lett. B 345, 441 (1995).

${ }^{7}$ J. Smoller and A. Wasserman, Phys. Rev. D 52, 5812 (1995).
} 
${ }^{8}$ J. Smoller, A. Wasserman, S.-T. Yau, and J. McLeod, Commun. Math. Phys. 143, 115 (1991).

${ }^{9}$ J. Smoller and A. Wasserman, Commun. Math. Phys. 151, 303 (1993).

${ }^{10}$ J. Smoller and A. Wasserman, J. Math. Phys. 36, 4301 (1995).

${ }^{11}$ P. Bizon, Phys. Rev. Lett. 61, 141-144 (1988).

${ }^{12}$ B. Carter, Phys. Lett. 21, 423 (1966). 